
implications for the deep Earth's interior

Alexander F. Goncharov ${ }^{1,2}$, Sergey S. Lobanov ${ }^{1,3}$, Xiaojing Tan ${ }^{1}$, Gregory T. Hohensee ${ }^{4}$, David G. Cahill ${ }^{4}$, Jung-Fu Lin ${ }^{5}$, Sylvia-Monique Thomas ${ }^{6}$, Takuo Okuchi ${ }^{7}$, and Naotaka Tomioka ${ }^{7}$.

${ }^{1}$ Geophysical Laboratory, Carnegie Institution of Washington, Washington DC

${ }^{2}$ Key Laboratory of Materials Physics, Institute of Solid State Physics, Chinese Academy of Sciences, Hefei, Anhui 230031, China

${ }^{3}$ V.S. Sobolev Institute of Geology and Mineralogy, SB RAS, Novosibirsk 630090, Russia.

${ }^{4}$ Department of Materials Science and Engineering and Materials Research Laboratory, University of Illinois, Urbana, IL.

${ }^{5}$ Department of Geological Sciences, Jackson School of Geosciences, The University of Texas at Austin, Austin, TX

${ }^{6}$ Department of Geoscience, University of Nevada Las Vegas, Las Vegas, NV

${ }^{7}$ Institute for Study of the Earth's Interior, Okayama University, Misasa, Tottori, Japan

Lattice thermal conductivity of ferropericlase and radiative thermal conductivity of iron bearing magnesium silicate perovskite (bridgmanite) - the major mineral of Earth's lower mantle- has been measured at room temperature up to 30 and $46 \mathrm{GPa}$, respectively, using time domain thermoreflectance and optical spectroscopy techniques in diamond anvil cells. The results provide new constraints for the pressure dependencies of the thermal conductivities of $\mathrm{Fe}$ bearing minerals. The lattice thermal conductivity of ferropericlase $\mathrm{Mg}_{0.9} \mathrm{Fe}_{0.1} \mathrm{O}$ is 5.7(6) W/(m*K) at ambient conditions, which is almost 10 times smaller than that of pure MgO; however, it increases with pressure much faster (6.1(7)\%/GPa vs 3.6(1)\%/GPa). The radiative conductivity of $\mathrm{Mg}_{0.94} \mathrm{Fe}_{0.06} \mathrm{SiO}_{3}$ bridgmanite single crystal agrees with previously determined values for powder samples at ambient pressure; it is almost pressure-independent in the investigated pressure range. Our results confirm the reduced radiative conductivity scenario for the Earth's lower mantle, while the assessment of the heat flow through the core-mantle boundary still requires in situ measurements at the relevant pressure-temperature conditions.

\title{
Introduction
}

The thermal conductivity $(k)$ of planetary materials is one of the key parameters for heat transport models within planetary interiors; it thus plays an important role in determining the temperature profile and energy balance of our planet (Lay et al., 2008). Furthermore, knowing the thermal conductivity of materials in the Earth's and other planetary interiors is critical for understanding the thermal history of the core and mantle and their dynamics. These are all of direct relevance for planetary accretion and differentiation processes (Stevenson, 1990), the time evolution of mantle and core temperatures (Stacey and Loper, 1984), and the generation of Earth's magnetic field (Stevenson, 2003).

Of the central questions regarding the thermal behavior of the planet is the magnitude of the heat flux across the core-mantle boundary (CMB) (Lay et al., 2008). The thermal boundary layer above the $\mathrm{CMB}$ regulates the rate of heat transfer between the core and mantle, providing one of the most important controls of thermal evolution 
and dynamics of the Earth (Labrosse, 2003; Labrosse et al., 2007; Stevenson, 2003). Knowledge of the depth-dependent thermal conductivity of the Earth's mantle would complement recent advances in mantle convection modeling, where a range of possible dynamic structures are predicted using estimated values for the thermal conductivity of the region (Dubuffet et al., 1999; Naliboff and Kellogg, 2006; Tosi et al., 2010). Current estimates of the thermal conductivity of mantle and core materials often rely on extrapolations of experimentally determined values at relatively low pressures and/or temperatures (P-T) (Hofmeister, 1999; Hofmeister, 2010; Hofmeister, 2014) and theoretical calculations (Cohen, 1998; de Koker, 2009, 2010; Dekura et al., 2013; Haigis et al., 2012; Stackhouse et al., 2010; Tang et al., 2014; Tang and Dong, 2009). Experimental data are largely limited in $P-T$ conditions of either high pressures or high temperatures alone (Chai et al., 1996; Dalton et al., 2013; Hsieh et al., 2009; Imada et al., 2014; Manthilake et al., 2011; Ohta et al., 2012; Osako and Ito, 1991) or are not sufficiently accurate due to assumptions needed in the modeling (Beck et al., 2007; Goncharov et al., 2010).

Other than our limited knowledge of the lattice thermal conductivity, the role of the radiative thermal conductivity in heat transport in the lower mantle remains controversial (Goncharov et al., 2008; Hofmeister, 2014; Hofmeister and Yuen, 2007; Keppler et al., 2008; Tang et al., 2014), because available estimates continue to rely on extrapolations and assumptions. Knowledge of the depth-dependent radiative thermal conductivity is important for assessment of the evolution of the mantle, such as generation and stability of thermo-chemical plumes in the lower mantle. At high temperature (ca $1000 \mathrm{~K}$ ), the radiative contribution to the thermal conductivity of mantle materials may increase substantially (Clark, 1956; Shankland et al., 1979). In situ measurements of the optical properties of mantle minerals at relevant high $P-T$ conditions will directly address these controversies.

Based on our previously developed technical methodology (Beck et al., 2007; Dalton et al., 2013; Goncharov et al., 2009; Goncharov et al., 2008; Goncharov et al., 2006; Goncharov et al., 2010), here we report on direct measurements of the lattice and radiative thermal conductivity of mantle minerals with extended compositional parameters under the relevant pressure conditions using optical spectroscopy and pulsed laser techniques in diamond anvil cells (DAC). A companion paper in the same volume (McWilliams et al., 2014) will be devoted to addressing the development of the flash laser heating technique, which combines pulsed and continuous heating methods. This technique is used for in situ measurements of the thermal conductivity of metals (e.g., $\mathrm{Fe})$ or minerals sandwiched by metallic layers at high $P-T$ conditions to better understand the thermal transport and temperature profile near the CMB.

\section{Experimental techniques}

Thermal conductivity measurements involve determination of heat fluxes through the examined material. This can be achieved either in the temporal or in the spatial domain depending on the nature and magnitude of the effects recorded. Thermal conductivity measurements at extreme $P-T$ conditions are challenging because the spatial scale that can be probed in the DAC cavity is very small, thereby making accurate measurements difficult. It is also difficult to extend the results of such measurements to the conditions of 
the Earth's interior, which has much larger space and time scales as well as different chemical and morphological characteristics than samples studied in the DAC.

The methodology for measuring the thermal conductivity in a DAC has been described in our previous publications (Beck et al., 2007; Dalton et al., 2013; Goncharov et al., 2006; Goncharov et al., 2010), and is thus not reiterated in this paper. Here and in the companion publication of (McWilliams et al., 2014) we focus on the discussion of the use of two new technical developments: (1) an updated flash heating method for measuring combined lattice and electrical thermal conductivity of metals, which uses a superposition of continuous and pulsed laser heating; (2) use of a supercontinuum laser light source method in optical spectroscopy of materials for derivation of the radiative conductivity.

Lattice thermal conductivity (or combined lattice and electrical conductivity in metals) measurements rely on temporal and/or spatial determination of the temperature gradient across the sample in transient and steady-state experiments, respectively. Since measuring temperature of the sample using a thermocouple attached to the heated sample in a DAC is practically problematic, the temperature in thermal conductivity experiments is usually obtained by thermoradiometry (Beck et al., 2007; Konôpková, 2011) or thermoreflectance methods (Hsieh et al., 2009; Yagi et al., 2011). The latter technique, which is based on the determination of very small changes in sample reflectivity due to changes in its temperature, is in principle more accurate, but it is also much more difficult to handle correctly, especially at high temperature conditions relevant to the deep Earth. On the other hand, the flash heating technique suffers from non-reproducibility and timedrifting due to chemical degradation of the sample (Goncharov et al., 2010). Timedomain thermoreflectance (TDTR) in a DAC using ultrafast laser pulses (cf. the technique with the same name in a $\mu$ s time scale of (Imada et al., 2014; Yagi et al., 2011)) arguably remains the most accurate method (Chen et al., 2011; Dalton et al., 2013). The drawback of this technique is the necessity to prepare samples with a laser transducer that has an ideal optical surface and is in good thermal contact with the material of interest. Measurements at high $P$ - $T$ conditions, especially using laser heating, remain a challenge because of the $P-T$ dependent opto-acoustic coupling and the thermal stability of the transducer. Moreover, the presence of the pressure medium with a thermal conductivity comparable to the sample can significantly influence the measurements, making the derived thermal conductivity less accurate. Below, we present preliminary TDTR measurements of $\mathrm{Mg}_{0.9} \mathrm{Fe}_{0.1} \mathrm{O}$ ferropericlase to $30 \mathrm{GPa}$ at $300 \mathrm{~K}$, which complement recently reported measurements of $\mathrm{MgO}$ to $60 \mathrm{GPa}$ (Dalton et al., 2013).

Determination of the radiative thermal conductivity is critically dependent on the optical properties of minerals (Clark, 1957). The optical system which we use for these studies is optimized for DAC operation and designed to avoid chromatic aberrations (due to the use of all-mirror optics) and to perform glitch-free measurements in a broad spectral range from near ultraviolet (UV) to mid infrared (IR). The inevitable chromatic aberrations due to the presence of diamond anvils in the optical system are small ( $\sim 4 \mathrm{um}$ for the spectral range probed) compared to the probed area dimensions ( $15 \mathrm{um})$. Moreover, these have been partially compensated by using the standard spectrum measured through the same diamond anvils.

The latest modification of the optical spectroscopy system includes the use of an offshelf turn-key supercontinuum light source for measurements in the spectral range from 
visible to near IR. This increases the signal-to-noise ratio, improves the spatial resolution up to submicron level, and enables measurements at high temperatures well above 1000 $140 \mathrm{~K}$ in the laser heated DAC due to the much larger instantaneous brightness (at least by 6 orders of magnitude compared to a conventional halogen/ $\mathrm{D}_{2}$ light source), which had been a problem previously.

\section{Lattice thermal conductivity of ferropericlase $\mathrm{Mg}_{0.9} \mathrm{Fe}_{0.1} \mathrm{O}$ to $30 \mathrm{GPa}$}

These measurements have been aimed at clarifying the dependence of the lattice thermal transport on $\mathrm{Fe}$ substitution in $\mathrm{MgO}$, especially the part which is responsible for the density dependence. Experimental and theoretical works undoubtedly show that mass substitution reduces the lattice thermal conductivity (Manthilake et al., 2011; Morton and Lewis, 1971), but the difference between a pure and a substituted material is expected to become smaller at high temperatures (Manthilake et al., 2011; Tang et al., 2014). However, the pressure (or volume) dependence of the thermal conductivity in such mass substituted materials remains uncertain as there is no experimental data, while theoretical calculations (Dalton et al., 2013) suggest a smaller pressure coefficient than that for the pure $\mathrm{MgO}$ endmember.

Ferropericlase samples of approximate $\mathrm{Mg}_{0.9} \mathrm{Fe}_{0.1} \mathrm{O}$ composition were synthesized in a gas-mixing furnace at high temperature and 1 atmosphere using $\mathrm{Mg}-\mathrm{Fe}$ interdiffusion between single-crystal $\mathrm{MgO}$ and pre-reacted $\mathrm{Mg}_{0.8} \mathrm{Fe}_{0.2} \mathrm{O}$ powder surrounding the crystal. Single crystals were polished to $15 \mu \mathrm{m}$ thickness using grinding wheels with a $3 \mu \mathrm{m}$ diamond grit, followed by $0.3 \mu \mathrm{m}$ alumina powder. The samples were heated to $1200 \mathrm{~K}$ for 15-30 minutes under vacuum to remove hydroxides and other volatile hydrocarbons from the polished surface (Dalton et al., 2013). One side of each sample was then coated with an $80 \mathrm{~nm} \mathrm{Al}$ film via magnetron sputtering.

The sample and a small ruby chip were loaded into the DAC with either Ar or $1 \mathrm{cSt}$ silicone oil (Sigma-Aldrich octamethyldisiloxane 98\%) as the pressure medium, so that the $\mathrm{Al}$ film was positioned between the $\mathrm{Mg}_{0.9} \mathrm{Fe}_{0.1} \mathrm{O}$ sample and the pressure-transmitting medium. The silicone oil has a smaller thermal conductivity smaller than Ar, which helps in determining the thermal conductivity of the sample more accurately. The thermal conductivity of $\mathrm{Mg}_{0.9} \mathrm{Fe}_{0.1} \mathrm{O}$ was measured at room temperature using the TDTR pumpprobe technique (Chen et al., 2011; Dalton et al., 2013; Hsieh et al., 2009), which determines the change in reflectivity of $\mathrm{Al}$ due to the temperature evolution caused by the incidence of an ultrashort ( $<200 \mathrm{fs})$ pump laser pulse. The change in reflectivity (proportional to temperature) is detected by a probe beam which is part of the same laser output of a mode-locked Ti:Sapphire oscillator ( $800 \mathrm{~nm}$ wavelength) but is time-delayed with respect to the pump beam. The probe pulses thereby monitor, as a function of time delay, the pump heating and subsequent cooling due to heat transfer to surrounding media. The pump and probe laser spot dimensions were approximately $12 \mu \mathrm{m}$ in diameter; since the thermal penetration depth of the experiment was about $1 \mu \mathrm{m}$, the heat transport was essentially one-dimensional. A Si photodiode and an RF lock-in amplifier were used to monitor the intensity of the in-phase $\left(\mathrm{V}_{\mathrm{in}}\right)$ and out-of-phase $\left(\mathrm{V}_{\text {out }}\right)$ signals

180 using the variation of the reflected probe beam intensity in response to the pump. The 181 thermal conductivity is determined as a best fit to a heat transfer model which was 182 extended to account for bi-directional heat flow into both the sample and pressure 
medium (Cahill, 2004) (Fig. 1a). The parameters of these calculations are similar to those reported by (Dalton et al., 2013).

The experiments have been performed on a free-standing sample and on the same
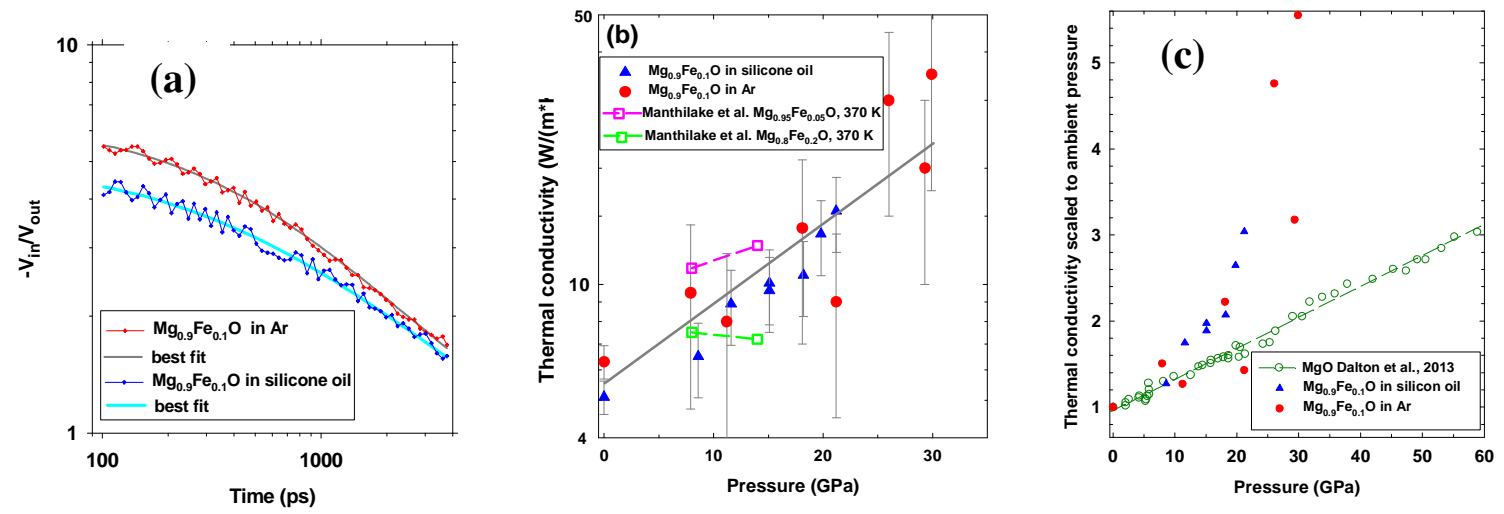
sample in the DAC under pressure. The former experiment determines the thermal effusivity much more accurately as the thermal transport from the heated Al layer is almost completely determined by the thermochemical properties of the sample (heat transport through the air is negligible). In the DAC, the results critically depend on the thermal conductivity of the pressure transmitting medium, which is strongly pressure dependent (Goncharov et al., 2012; Tretiakov and Scandolo, 2004). Our derived thermal conductivity values of $\mathrm{Mg}_{0.9} \mathrm{Fe}_{0.1} \mathrm{O}$ are in fair agreement with previous measurements in a large press (Manthilake et al., 2011) (Fig. 1b). However, the pressure dependence, which we determined up to $30 \mathrm{GPa}$, is much higher than that inferred from two high-pressure points of (Manthilake et al., 2011) and also much higher than the results of the calculations based on the Debye-Callaway model (Dalton et al., 2013) (Fig. 1c). Further experimental efforts involving different pressure media at higher pressures and high temperatures are needed to assess the effect of the mass disorder on the thermal conductivity in mantle minerals at relevant $P-T$ conditions of the Earth's lower mantle.

Fig. 1. Lattice thermal conductivity of the $\mathrm{Mg}_{0.9} \mathrm{Fe}_{0.1} \mathrm{O}$ single-crystal. (a) Ratio $\left(-\mathrm{V}_{\text {in }} / \mathrm{V}_{\text {out }}\right)$ signal as a function of time delay for DAC TDTR experiments in Ar and silicone oil pressure media at 8.0 and 8.6 GPa, respectively, along with fits to the spectra; (b) The thermal conductivity of $\mathrm{Mg}_{0.9} \mathrm{Fe}_{0.1} \mathrm{O}$ as a function of pressure determined in this work using Ar and silicone oil media in comparison with the results obtained in a large press using the Ångström method (Manthilake et al., 2011) at $370 \mathrm{~K}$; (c) The ratios of the thermal conductivity at high pressures to that at ambient pressure. Our results for $\mathrm{Mg}_{0.9} \mathrm{Fe}_{0.1} \mathrm{O}$ are compared to the results for pure $\mathrm{MgO}$ from (Dalton et al., 2013).

\section{Radiative thermal conductivity of $\mathrm{Mg}_{0.94} \mathrm{Fe}_{0.06} \mathrm{SiO}_{3}$ bridgmanite to $47 \mathrm{GPa}$}

The optical absorption measurements reported here address the existing controversy between published experimental results (Goncharov et al., 2008; Keppler et al., 2008) and estimations based on rough approximations (Hofmeister, 2014). Bridgmanite is believed to be the most abundant mineral in the Earth's lower mantle, thus knowledge of the optical conductivity of this material is of primary importance to our understanding of the thermal transport behavior of the most voluminous layer of the planet. 
Here we study single crystals of $\mathrm{Mg}_{(1-\mathrm{x})} \mathrm{Fe}_{\mathrm{x}} \mathrm{SiO}_{3}(\mathrm{x}=0.06$ ) synthesized using the Kawai Apparatus at approximately $24 \mathrm{GPa}$ and $2100 \mathrm{~K}$ for $6 \mathrm{hrs}$ in a Pt capsule at the University of Okayama at Misasa, Japan (Okuchi et al., 2015; Shatskiy et al., 2007). A mixture of $\mathrm{SiO}_{2},{ }^{57} \mathrm{Fe}$-enriched $(\mathrm{Mg}, \mathrm{Fe}) \mathrm{O}$, and brucite was used as the starting material and was loaded into a Pt capsule; the brucite was used to provide a water-rich environment in the sample chamber in order to enhance the crystal growth during the experiment (Shatskiy et al., 2007). The crystal structure, chemical composition, and iron oxidation states of the bridgmanite single crystal were carefully characterized using $\mathrm{X}$ ray diffraction, electron microscopy, and Mössbauer spectroscopy, showing a chemical composition of $\mathrm{Mg}_{0.94} \mathrm{Fe}_{0.06} \mathrm{SiO}_{3}$ with $45 \%$ of the total $\mathrm{Fe}$ in the $\mathrm{Fe}^{3+}$ state. Single-crystal bridgmanite samples of approximately $40-100 \mu \mathrm{m}$ in diameter and $30 \mu \mathrm{m}$ thickness were polished down to an approximate thickness of $25 \mu \mathrm{m}$ using $1 \mu \mathrm{m} 3 \mathrm{M}$ diamond lapping films, and water as lubricant; the polishing process also assured the removal of any potential contamination on the sample surface due to high-temperature quenching in the sample synthesis process. Since bridgmanite samples are known to become unstable and amorphize upon heating or mechanical grinding, the crystallinity of the samples after polishing was further confirmed using the electron microscopy and Raman spectroscopy analyses.

The bridgmanite crystal of approximately $40 \times 40 \mu \mathrm{m}^{2}$ dimensions and $25 \mu \mathrm{m}$ in thickness was loaded in a cavity of a symmetric DAC along with the Ar pressure transmitting medium and ruby pressure calibrant (Fig. 2a). The sample thickness has been measured before the Ar gas loading using the interference fringes method (Dewaele et al., 2003). Since the refractive index of bridgmanite and its pressure dependence are not well known, we adopted the pressure independent value of refractive index $n=1.8$ as suggested by Keppler et al., (2008). We have monitored the DAC cavity thickness using measurements of the interference fringes in the reflectivity spectra of the sample cavity away from the sample; the pressure dependent refractive index of Ar has been used (Grimsditch et al., 1986) for these measurements. At $24 \mathrm{GPa}$, the thickness of the sample cavity in the DAC approached the sample thickness (determined from the bridgmanite equation of state (Fiquet et al., 1998)) such that the sample was bridged by the diamond anvils at higher pressures. No qualitative change in the optical absorption spectra has been detected after the bridging on the pressure release from $46 \mathrm{GPa}$ to $37 \mathrm{GPa}$, at which point we ended the experiment. The sample thickness above the bridging point was determined from the thickness of the sample cavity in the DAC. The sample was optically transparent without any visible optical defects in the measuring area up to the highest pressure of $46 \mathrm{GPa}$ (Fig. 2a) (cf. Hofmeister, 2014; Keppler et al., 2008). Also, our near IR transmission spectra did not show any sign of $\mathrm{OH} / \mathrm{H}_{2} \mathrm{O}$ absorption bands at any pressure, suggesting the absence of water at the wt. ppm level.

We have determined the optical absorption coefficient of the bridgmanite single crystal using a commonly adopted procedure (e.g., Goncharov et al., 2010), which includes measuring the reference transmission spectra through the Ar transmitting medium and through the sample. The reflectivity losses have been taken into account for both the reference and sample spectra using the pressure dependent refractive indices of the $\mathrm{Ar}$ medium (Grimsditch et al., 1986) and the assumed refractive indices of bridgmanite $\left(n_{b}=1.8\right)$ and diamond $\left(n_{d}=2.4\right)$. Our estimation using the Gladstone-Dale equation shows that the refractive index of bridgmanite is expected to change by less than 
$4 \%$ from 0 to $46 \mathrm{GPa}$. The change in refractive index of diamond is also very small, less than 2\% (Balzaretti and da Jornada, 1996). These variations will have no essential effect on the reflectivity correction (which remains negligible) and thus no effect on the results presented in Fig. 2(c). The pressure dependence of the absorption coefficient is shown in Fig. 2b. Consistent with our previous work (Goncharov et al., 2008), the absorption coefficient of the sample slightly increases with increasing pressure, resulting in a small decline in the radiative conductivity at higher pressure. The radiative conductivity has been determined as $k_{R}=\frac{16 n^{2} \sigma T^{3}}{3 \alpha_{R}}$, where $\alpha_{R}$ is the Rosseland mean absorption coefficient

\section{(a)}

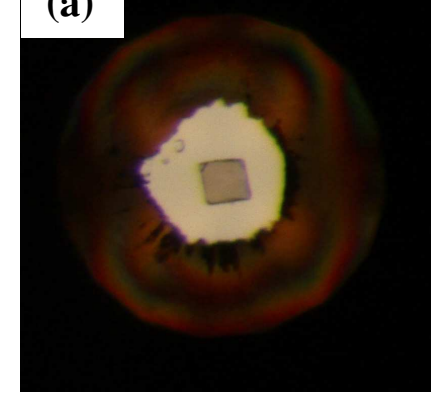

(b)

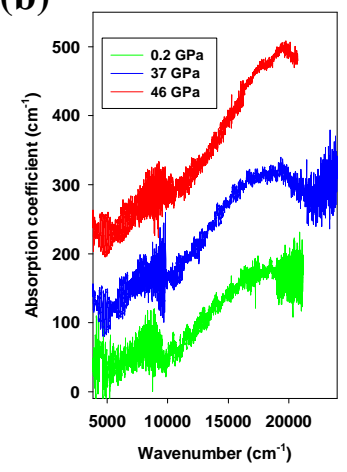

(c)

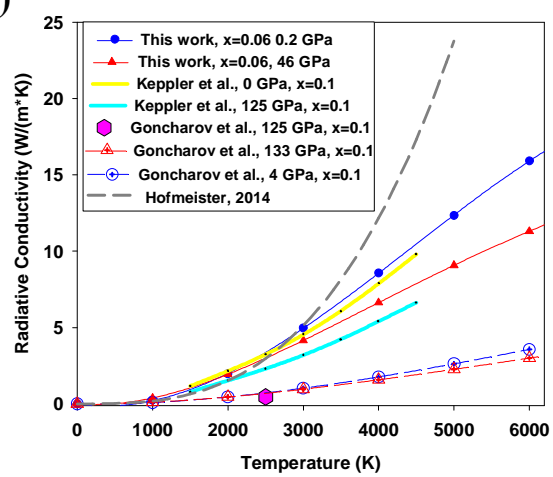

Fig. 2. Radiative thermal conductivity of single-crystal bridgmanite $\mathrm{Mg}_{0.94} \mathrm{Fe}_{0.06} \mathrm{SiO}_{3}$. (a) Representative microphotograph at $37 \mathrm{GPa}$. The sample cavity was about $120 \mu \mathrm{m}$ in diameter while the probed sample area was about $15 \mu \mathrm{m}$ in diameter. We note that the sample chamber geometry allowed sufficient space to conduct the transmission measurements through the sample as well as through the sample cavity filled with Ar; (b) Optical absorption spectra of the bridgmanite sample at high pressures. The absorption coefficient is calculated using $\alpha=A * \ln 10 / d$, where $A=\log _{10}\left(I_{0} / I\right), I_{0}$ and $I$ are the reference and sample signals respectively, and $d$ is the sample thickness which is determined as described in detail in the text. The reflectivity corrections for $I$ and $I_{0}$ have been made using the refractive indices of diamond, Ar, and bridgmanite. The spectra are offset with a 100 $\mathbf{~ c m}^{-1}$ increment for clarity; (c) Radiative thermal conductivity of the bridgmanite sample as a function of temperature. The values have been calculated using the method described by (Keppler et al., 2008). Our results for the single-crystal bridgmanite with $\mathrm{x}=\mathbf{0 . 0 6}$ are compared with previous studies (Goncharov et al., 2008; Keppler et al., 2008) investigating bridgmanite samples with $\mathrm{x}=\mathbf{0 . 1}$. The original optical absorption data of (Goncharov et al., 2008) have been processed using the same formalism to determine the radiative conductivity as a function of temperature.

The absorption coefficient of our sample is smaller than previously reported values, which is due to the smaller Fe content (6\% vs $10 \%$ ). This leads to noisier spectra, however major spectral features are sufficiently resolved. Our results at $0.2 \mathrm{GPa}$ agree 
composition (Keppler et al., 2008) taken at ambient conditions. Similar to previous observations in polycrystalline materials (Goncharov et al., 2008; Keppler et al., 2008), the absorption spectra of the bridgmanite single crystal show a complex absorption edge with several characteristic bands. In the near IR region at $\sim 8000 \mathrm{~cm}^{-1}$, the absorption band arising from the crystal-field transition of $\mathrm{Fe}^{2+}$ can be clearly identified. However, unlike our previous observations (Goncharov et al., 2008), we could not detect any intensity change of this band with pressure, which may be due to the poorer signal-tonoise ratio of the spectra. The intervalence band and/or the UV absorption edge (which was difficult to measure, but was still detectable in our best spectra) slowly increases in intensity with pressure. This observation is in qualitative agreement with our previous results (Goncharov et al., 2008). Overall, the single-crystal spectra are in good agreement with our previous work on polycrystalline samples. The presence of the UV edge in the single-crystal bridgmanite with good optical properties rules out the possibility that the edge is due to sample imperfections or grain boundary scattering (Hofmeister and Yuen, 2007), and it also confirms our previous assignment of this absorption edge to the $\mathrm{O}^{2-}$ $\mathrm{Fe}^{3+}$ charge transfer gap (Goncharov et al., 2008).

The derived radiative conductivities of $\mathrm{Mg}_{0.94} \mathrm{Fe}_{0.06} \mathrm{SiO}_{3}$ (Fig. 2c) show substantially larger values compared to those reported in our previous work on polycrystalline $\mathrm{Mg}_{0.9} \mathrm{Fe}_{0.1} \mathrm{SiO}_{3}$ (Goncharov et al., 2008). We attribute this deviation to the difference in the Fe content of the samples. Bridgmanite with a nominal Fe composition of $\mathrm{x}=0.1$ reveals stronger absorption, and consequently is expected to conduct less radiative heat. It should be noted that a substantial discrepancy exists between these data and those reported by (Keppler et al., 2008) on material with the same nominal Fe composition $(x=0.1)$, while the results of both groups agree on the mineral with $x=0.05-0.06$ measured at ambient pressure. We attribute this discrepancy to either the difference in the $\mathrm{Fe}^{3+}$ concentration, which can affect the intervalence transitions, or the sample thickness used in the measurements of (Keppler et al., 2008), since a possible sample bridging in their work was not documented.

It is interesting that both the absorption coefficient and the radiative conductivity change nonlinearly with the Fe composition. We suggest that a substantial contribution to the visible and near IR absorption arises from the $\mathrm{Fe}^{2+}-\mathrm{Fe}^{3+}$ intervalence transitions, which require these ions to be adjacent to each other; the probability of this event increases with increasing the $\mathrm{Fe}$ concentration $(\mathrm{x})$ as $\mathrm{x}^{2}$. As expected, the radiative conductivity increases with temperature as $\mathrm{T}^{3}$ (e.g., Hofmeister, 2014) only at temperatures below approximately $2000 \mathrm{~K}$. At higher temperatures, there is a large deviation from this behavior to smaller values due to the increased contribution from the intervalence transitions and the charge-transfer gap. Moreover, our high-pressure measurements show a decrease in the radiative conductivity due to an increase of the absorption coefficient. We conclude that these direct and accurate measurements of the optical properties of a well-characterized mantle bridgmanite sample are important for assessment of the radiative conductivity at relevant $P-T$ conditions. Further works in this direction should include such measurements at high temperatures representative for the deep interior. The use of a supercontinuum light source enables such measurements in the most critical spectral range between 400 and $2400 \mathrm{~nm}\left(4200-25000 \mathrm{~cm}^{-1}\right)$.

\section{Conclusions}


We present updated experimental results on lattice and radiative thermal conductivity of the lower-mantle minerals ferropericlase and bridgmanite. These studies require a full understanding of all the important aspects that could affect the thermal conductivity at relevant $P$-T environments of the mantle. Here we shed some light on the consistency of recent measurements between different groups using various experimental techniques and studying diverse samples. Future studies should use these results as benchmarks in their experiments and models, which will be aiming to solve unresolved issues with mass substitution, temperature dependencies, and sample morphology.

\section{Acknowledgements}

We thank Brent Grocholski from Smithsonian Institution for providing the ferropericlase sample. We acknowledge support from the NSF EAR, NSF EAR/IF, Army Research Office, DARPA and EFREE, a BES-EFRC center at Carnegie. S. S. L. was partly supported by the Ministry of Education and Science of Russian Federation (Grant No. 14.B25.31.0032). G. T. H. and D. G. C. acknowledge support from the Carnegie-DOE Alliance Center (CDAC). SMT acknowledges support from NSF EAR grants 1215957 and 1417274. J. F. L. acknowledges financial support from NSF Earth Sciences (EAR0838221 and EAR-1446946), Deep Carbon Observatory, and the Visiting Professorship Program at the Institute for the Study of the Earth's Interior of the University of Okayama at Misasa.

\section{Bibliography}

Balzaretti, N.M., da Jornada, J.A.H., 1996. Pressure dependence of the refractive index of diamond, cubic silicon carbide and cubic boron nitride. Solid State Communications 99, 943-948.

Beck, P., Goncharov, A.F., Struzhkin, V.V., Militzer, B., Mao, H.K., Hemley, R.J., 2007. Measurement of thermal diffusivity at high pressure using a transient heating technique. Appl Phys Lett 91, 181914.

Cahill, D.G., 2004. Analysis of heat flow in layered structures for time-domain thermoreflectance. Review of Scientific Instruments 75, 5119-5122.

Chai, M., Brown, J.M., Slutsky, L.J., 1996. Thermal diffusivity of mantle minerals. Phys Chem Miner 23, 470-475.

Chen, B., Hsieh, W.-P., Cahill, D.G., Trinkle, D.R., Li, J., 2011. Thermal conductivity of compressed $\mathrm{H} 2 \mathrm{O}$ to $22 \mathrm{GPa}$ : A test of the Leibfried-Schlömann equation. Phys Rev B 83, 132301.

Clark, S.P., 1956. Effect of Radiative Transfer on Temperatures in the Earth. Geol Soc Am Bull 67, 1123-1124.

Clark, S.P., 1957. Radiative transfer in the Earth's mantle. Transactions, American Geophysical Union 33, 931-938.

Cohen, R.E., 1998. Thermal conductivity of $\mathrm{MgO}$ at high pressures, in: Nakahara, M. (Ed.), Proceedings of International Conference -- AIRAPT-16 and HPCJ-38 -- on High Pressure Science and Technology (Rev. High Pressure Sci. Tech.). Japan Society of High Pressure Science and Technology, Kyoto, pp. 160-161.

Dalton, D.A., Hsieh, W.-P., Hohensee, G.T., Cahill, D.G., Goncharov, A.F., 2013. Effect of Mass Disorder on the Lattice Thermal Conductivity of $\mathrm{MgO}$ Periclase Under Pressure: Implication for the Deep Earth Heat Flow. Physics Reports 3, 2400. 
de Koker, N., 2009. Thermal Conductivity of MgO Periclase from Equilibrium First Principles Molecular Dynamics. Physical Review Letters 103, 125902.

de Koker, N., 2010. Thermal conductivity of $\mathrm{MgO}$ periclase at high pressure: Implications for the D " region. Earth and Planetary Science Letters 292, 392-398. Dekura, H., Tsuchiya, T., Tsuchiya, J., 2013. Ab initio Lattice Thermal Conductivity of $\mathrm{MgSiO} 3$ Perovskite as Found in Earth's Lower Mantle. Physical Review Letters 110, 025904.

Dewaele, A., Eggert, J.H., Loubeyre, P., Le Toullec, R., 2003. Measurement of refractive index and equation of state in dense $\mathrm{He}, \mathrm{H} 2, \mathrm{H} 2 \mathrm{O}$, and $\mathrm{Ne}$ under high pressure in a diamond anvil cell. Phys Rev B 67, 094112.

Dubuffet, F., Yuen, D.A., Rabinowicz, M., 1999. Effects of a realistic mantle thermal conductivity on the patterns of 3-D convection. Earth and Planetary Science Letters 171, 401-409.

Fiquet, G., Andrault, D., Dewaele, A., Charpin, T., Kunz, M., Haüsermann, D., 1998. PV-T equation of state of $\mathrm{MgSiO} 3$ perovskite. Phys Earth Planet In 105, 21-31. Goncharov, A.F., Beck, P., Struzhkin, V.V., Haugen, B.D., Jacobsen, S.D., 2009.

Thermal conductivity of lower-mantle minerals. Phys Earth Planet Interior 174, 24-32. Goncharov, A.F., Haugen, B.D., Struzhkin, V.V., Beck, P., Jacobsen, S.D., 2008. Radiative conductivity in the Earth's lower mantle. Nature 456, 231-234. Goncharov, A.F., Struzhkin, V.V., Jacobsen, S.D., 2006. Reduced radiative conductivity of low-spin (Mg,Fe)O in the lower mantle. Science 312, 1205-1208.

Goncharov, A.F., Struzhkin, V.V., Montoya, J.A., Kharlamova, S., Kundargi, R., Siebert, J., Badro, J., Antonangeli, D., Ryerson, F.J., Mao, W., 2010. Effect of composition, structure, and spin state on the thermal conductivity of the Earth's lower mantle. Phys Earth Planet In 180, 148-153.

Goncharov, A.F., Wong, M.L., Dalton, D.A., Ojwang, J., Struzhkin, V., Konopkova, Z., Lazor, P., 2012. Thermal conductivity of argon at high pressures and high temperatures. J. Appl. Phys. 111, 112609.

Grimsditch, M., Loubeyre, P., Polian, A., 1986. Brillouin scattering and three-body forces in argon at high pressures. Phys Rev B 33, 7192-7200.

Haigis, V., Salanne, M., Jahn, S., 2012. Thermal conductivity of MgO, MgSiO3 perovskite and post-perovskite in the Earth's deep mantle. Earth and Planetary Science Letters 355-356, 102-108.

Hofmeister, A.M., 1999. Mantle values of thermal conductivity and the geotherm from phonon lifetimes (vol 283, pg 1699, 1999). Science 284, 264-264.

Hofmeister, A.M., 2010. Scale aspects of heat transport in the diamond anvil cell, in spectroscopic modeling, and in Earth's mantle: Implications for secular cooling. Phys. Earth Planet. Inter. 180, 138-147.

Hofmeister, A.M., 2014. Thermodynamic and optical thickness corrections to diffusive radiative transfer formulations with application to planetary interiors. Geophys Res Lett 41, 3074-3080.

Hofmeister, A.M., Yuen, D.A., 2007. Critical phenomena in thermal conductivity: Implications for lower mantle dynamics. J Geodyn 44, 186-199. Hsieh, W.-P., Chen, B., Li, J., Keblinski, P., Cahill, D.G., 2009. Pressure tuning of the thermal conductivity of the layered muscovite crystal. Phys Rev B 80, 180302-(180301180304)(R). 
Imada, S., Ohta, K., Yagi, T., Hirose, K., Yoshida, H., Nagahara, H., 2014. Measurements of lattice thermal conductivity of $\mathrm{MgO}$ to core-mantle boundary pressures. 462 Geophys Res Lett 41, 4542-4547.

463 Keppler, H., Dubrovinsky, L.S., Narygina, O., Kantor, I., 2008. Optical Absorption and 464 Radiative Thermal Conductivity of Silicate Perovskite to 125 Gigapascals. Science 322, 465 1529-1532.

466 Konôpková, Z., Lazor, P., Goncharov, A. F., Struzhkin, V. V., , 2011. Thermal

467 conductivity of hcp iron at high pressure and temperature, . High Pressure Research 31, $468 \quad 228-236$.

469 Labrosse, S., 2003. Thermal and magnetic evolution of the Earth's core. Phys. Earth 470 Planet. Inter. 140, 127-143.

471 Labrosse, S., Hernlund, J.W., Coltice, N., 2007. A crystallizing dense magma ocean at 472 the base of the Earth's mantle. Nature 450, 866-869.

473 Lay, T., Hernlund, J., Buffett, B.A., 2008. Core-mantle boundary heat flow. Nat Geosci $474 \quad 1,25-32$.

475 Manthilake, G.M., de Koker, N., Frost, D.J., McCammon, C.A., 2011. Lattice thermal 476 conductivity of lower mantle minerals and heat flux from Earth's core. Proceedings of the 477 National Academy of Sciences 108, 17901-17904.

478 McWilliams, R.S., Konopkova, Z., Goncharov, A.F., 2014. Thermal conductivity of metals measured in the diamond anvil cell at high pressures using a combination of pulsed and continuous laser heating technique.

Morton, I.P., Lewis, M.F., 1971. Effect of Iron Impurities on the Thermal Conductivity of Magnesium Oxide Single Crystals below Room Temperature. Phys Rev B 3, 552-559. Naliboff, J.B., Kellogg, L.H., 2006. Dynamic effects of a step-wise increase in thermal conductivity and viscosity in the lowermost mantle. Geophys Res Lett 33, L12S09-(1114).

Ohta, K., Yagi, T., Taketoshi, N., Hirose, K., Komabayashi, T., Baba, T., Ohishi, Y., Hernlund, J., 2012. Lattice thermal conductivity of MgSiO3 perovskite and postperovskite at the core-mantle boundary. Earth and Planetary Science Letters 349-350, 109-115.

Okuchi, T., Purevjav, N., Tomioka, N., Lin, J.F., Kuribayashi, T., Schoneveld, L., Hwang, H., Sakamoto, N., Kawasaki, N., Yurimoto, H., 2015. Synthesis of large and homogeneous single crystals of water-bearing minerals by slow cooling at deep-mantle pressures. American Mineralogist. doi:10.2138/am-2015-5237

Osako, M., Ito, E., 1991. Thermal-Diffusivity of Mgsio3 Perovskite. Geophys Res Lett 18, 239-242.

Planck, M., 1914. The Theory of Heat Radiation, translated by M. Masius, . P. Blakiston's, Philadelphia.

Shankland, T.J., Nitsan, U., Duba, A.G., 1979. Optical-Absorption and Radiative HeatTransport in Olivine at High-Temperature. J Geophys Res 84, 1603-1610. Shatskiy, A., Fukui, H., Matsuzaki, T., Shinoda, K., Yoneda, A., Yamazaki, D., Ito, E., Katsura, T., 2007. Growth of large $(1 \mathrm{~mm}) \mathrm{MgSiO} 3$ perovskite single crystals: A thermal gradient method at ultrahigh pressure. American Mineralogist 92, 1744-1749.

504 Planet In 36, 99-115. 
505 Stackhouse, S., Stixrude, L., Karki, B.B., 2010. Thermal Conductivity of Periclase

$506(\mathrm{MgO})$ from First Principles. Physical Review Letters 104, 208501.

507 Stevenson, D.J., 1990. Fluid dynamics of core formation, in: Newsom, H.E.J., J.E. (Ed.),

508 Origin of the Earth. Oxford Univ Press., Oxford, pp. 231-250.

509 Stevenson, D.J., 2003. Planetary magnetic fields. Earth Planet. Sci. Lett. 208, 1-11.

510 Tang, X., Ntam, M.C., Dong, J., Rainey, E.S.G., Kavner, A., 2014. The thermal

511 conductivity of Earth's lower mantle. Geophys Res Lett 41, 2746-2752.

512 Tang, X.L., Dong, J.J., 2009. Pressure dependence of harmonic and anharmonic lattice

513 dynamics in MgO: A first-principles calculation and implications for lattice thermal

514 conductivity. Phys Earth Planet In 174, 33-38.

515 Thomas, S.-M., Bina, C.R., Jacobsen, S.D., Goncharov, A.F., 2012. Radiative heat

516 transfer in a hydrous mantle transition zone. Earth and Planetary Science Letters 357-358

$517 \quad 130-136$.

518 Tosi, N., Yuen, D.A., Cadek, O., 2010. Dynamical Consequences in the Lower Mantle

519 with the Post-Perovskite Phase Change and Strongly Depth-Dependent Thermodynamic

520 and Transport Properties. Earth and Planetary Science Letters 298, 229-243.

521 Tretiakov, K.V., Scandolo, S., 2004. Thermal conductivity of solid argon at high pressure

522 and high temperature: A molecular dynamics study. The Journal of Chemical Physics

523 121, 11177-11182.

524 Yagi, T., Ohta, K., Kobayashi, K., Taketoshi, N., Hirose, K., Baba, T., 2011. Thermal

525 diffusivity measurement in a diamond anvil cell using a light pulse thermoreflectance

526 technique. Measurement Science and Technology 22, 024011.

527

528 
Fig1a

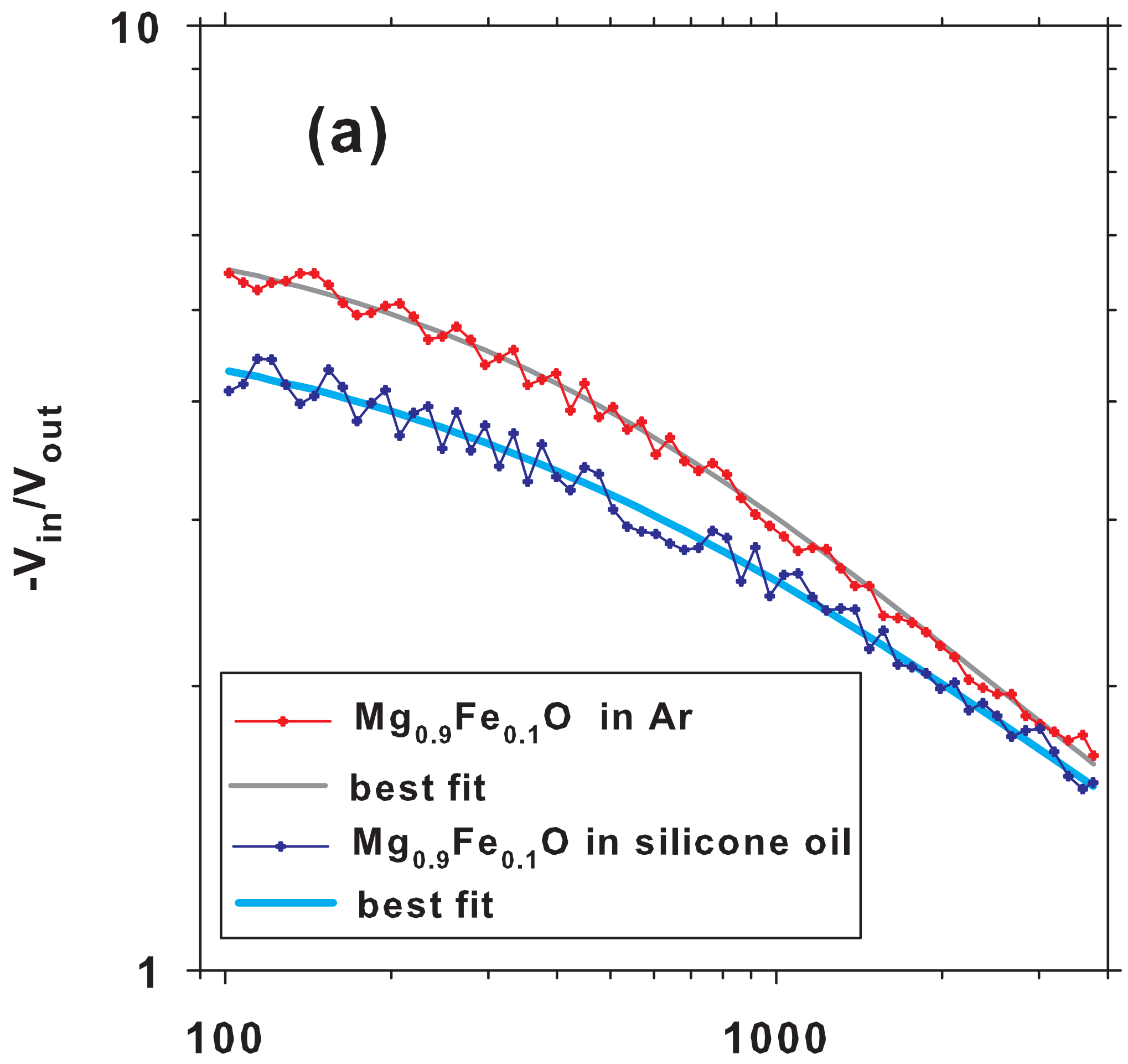

Time (ps) 
Fig1bM

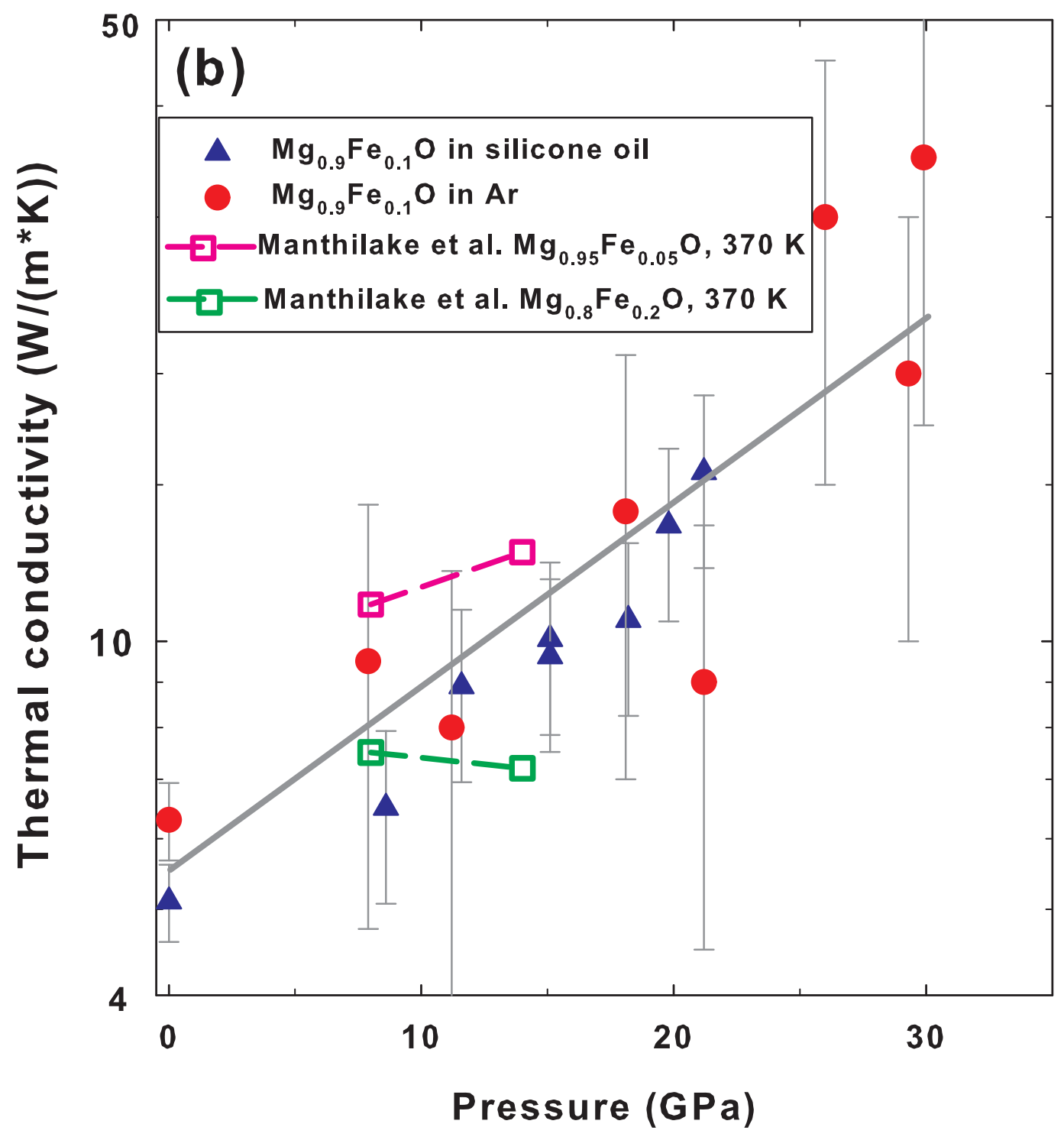


Fig1c

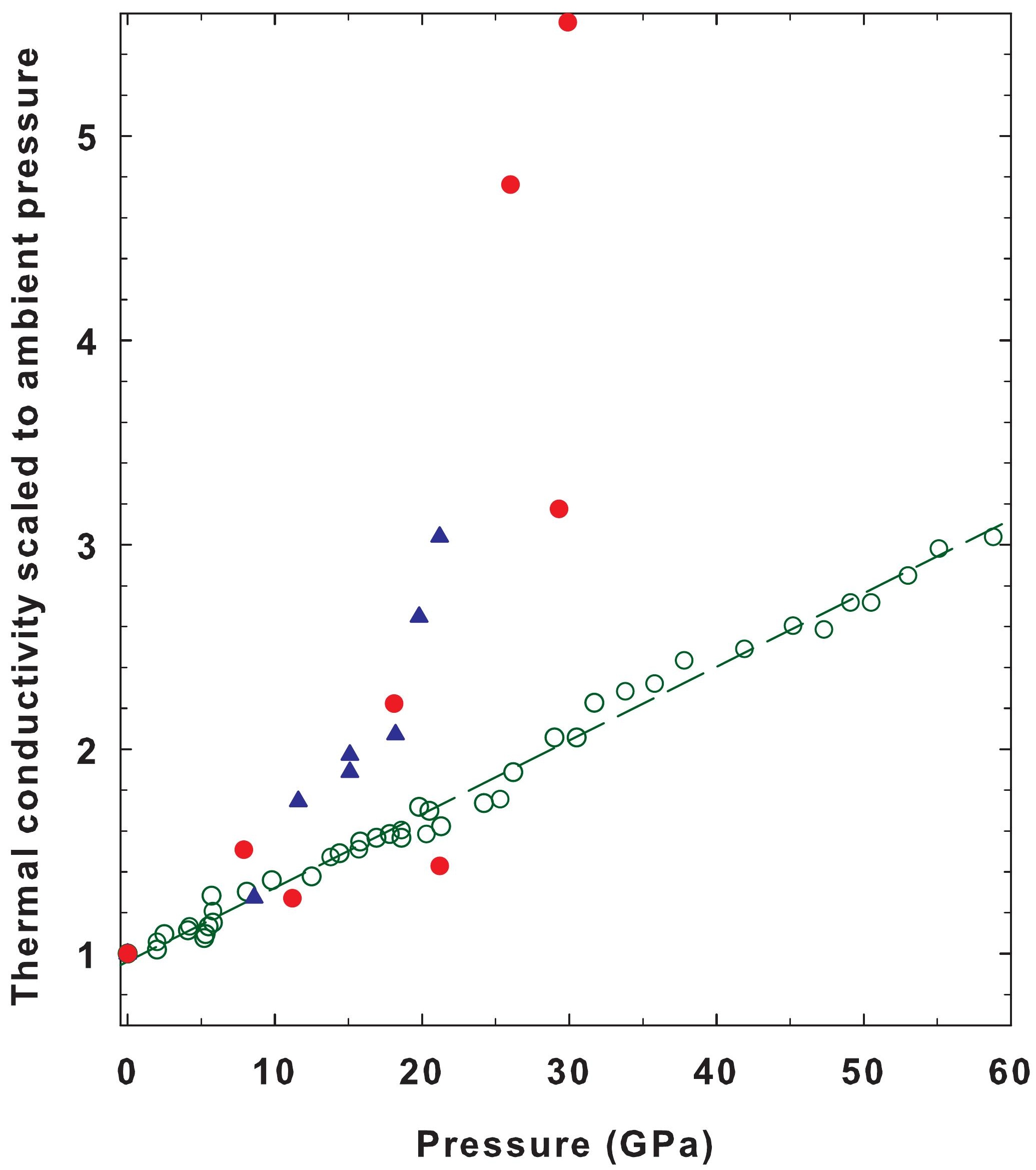


Fig2a

(a) 
Fig2b

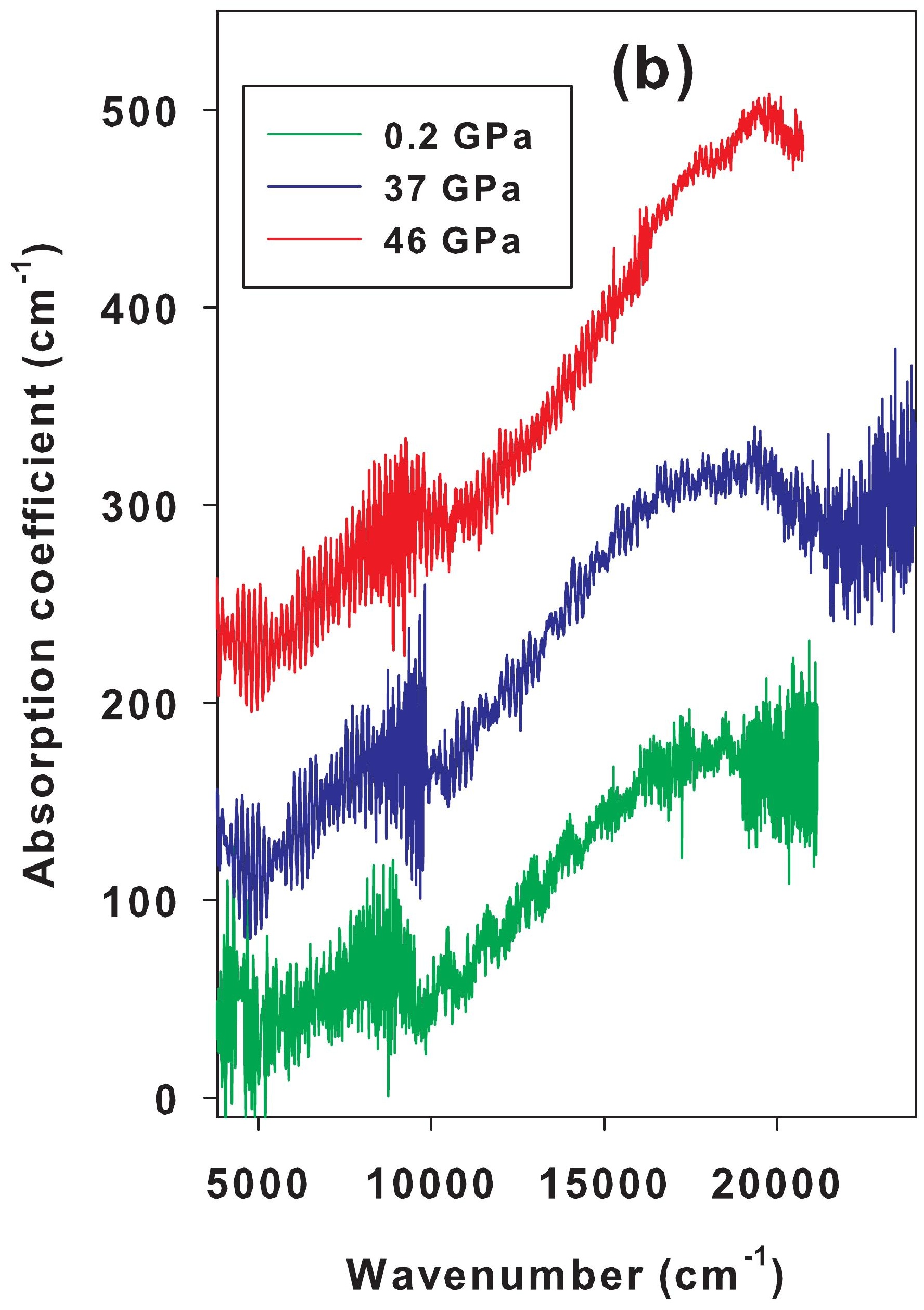


Fig2c

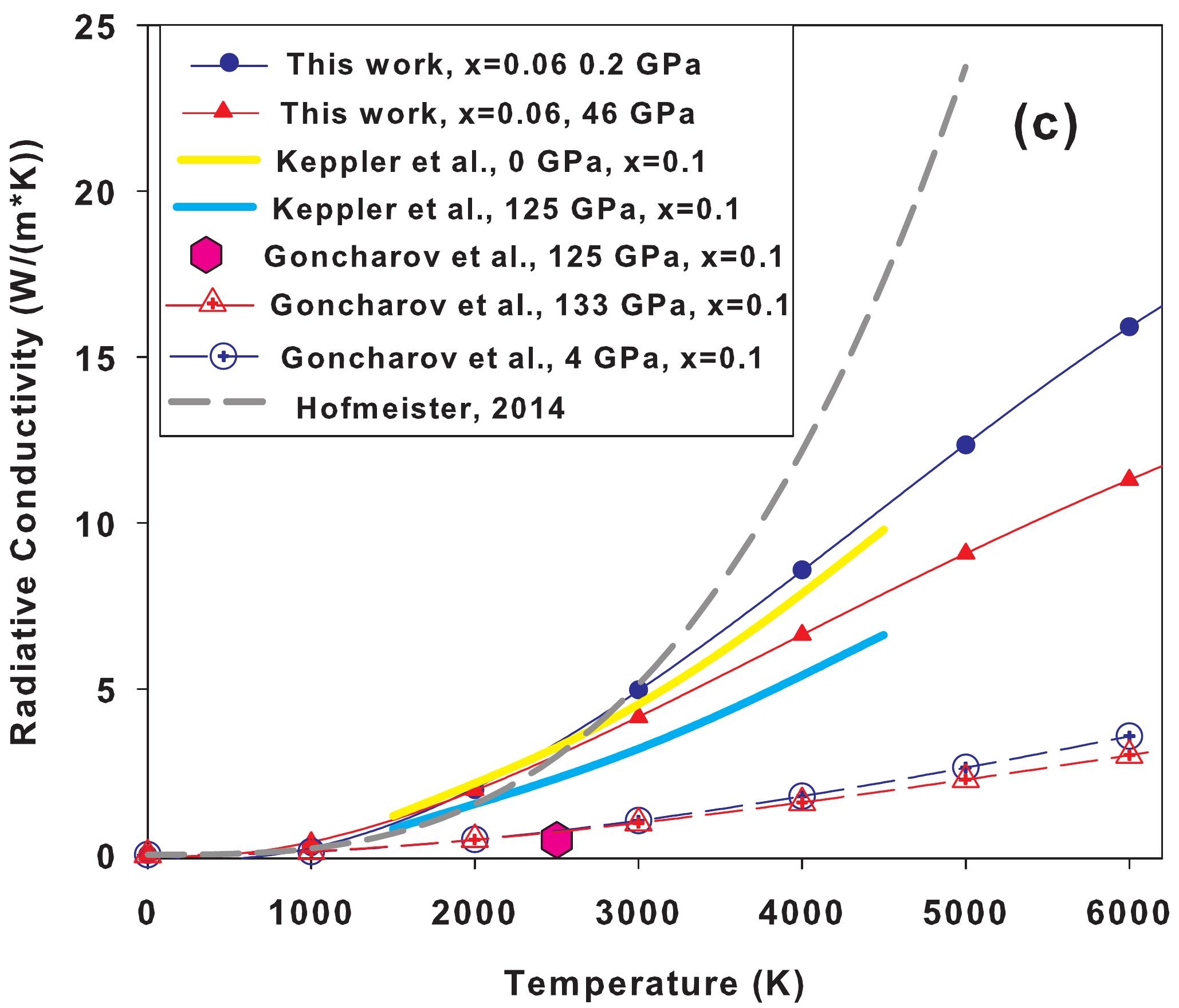

